\title{
Visual Perception of Incomplete Human-Like Body Postures: A Near-Infrared Spectroscopy Study
}

\author{
Hiroshi Ito' ${ }^{1}$, Hirofumi Saito ${ }^{2 *}$, Misato 0i², Tomoko Shiraishi' ${ }^{3}$, Shuang Meng², \\ Victor Alberto Palacios ${ }^{2}$ \\ ${ }^{1}$ Department of Psychology, Faculty of Letters, Aichi University, Toyohashi, Japan \\ ${ }^{2}$ Department of Cognitive Informatics, Graduate School of Information Science, Nagoya University, Nagoya, Japan \\ ${ }^{3}$ Department of Nursing, College of Life and Health Sciences, Chubu University, Kasugai, Japan \\ Email: saito@is.nagoya-u.ac.jp
}

Received 25 September 2014; revised 10 November 2014; accepted 26 November 2014

Copyright (C) 2014 by authors and Scientific Research Publishing Inc.

This work is licensed under the Creative Commons Attribution International License (CC BY).

http://creativecommons.org/licenses/by/4.0/

(c) (i) Open Access

\begin{abstract}
Previous studies have reported that the mirror neuron system plays a crucial role in social cognition. We examined whether the higher-order cognitive functions are involved in the activations in the mirror neuron area when we perceive simplified pseudo-postures. We measured 14 participants' brain activation during the posture-recognition task using near-infrared spectroscopy. The participants' task was to observe five sequentially presented target pseudo-postures and judge whether a test pseudo-posture was identical to one of the preceding five target pseudo-postures. The results in the majority of participants $(n=10 / 14)$ revealed that the activity in the inferior frontal mirror neuron area is modulated by perception of human-likeness, but not in the remaining four participants $(n=4 / 14)$. These results suggest that the degree of the activation of higherorder cognitive functions, which may be engaged in the inhibitory and/or facilitative processing of human body or bodily movement, leads to the distinctive activities in the inferior frontal mirror neuron area.
\end{abstract}

\section{Keywords}

Near-Infrared Spectroscopy (NIRS), Mirror Neuron System, Object Perception, Human Body

\section{Introduction}

Previous studies have reported that when we observe somebody else executing an action, many areas of our own

${ }^{*}$ Corresponding author.

How to cite this paper: Ito, H., Saito, H., Oi, M., Shiraishi, T., Meng, S. and Palacios, V.A. (2014) Visual Perception of Incomplete Human-Like Body Postures: A Near-Infrared Spectroscopy Study. Journal of Behavioral and Brain Science, 4, 544-558. http://dx.doi.org/10.4236/jbbs.2014.412053 
motor systems are activated. Functional magnetic resonance imaging (fMRI) studies have reported such activations in the ventral and dorsal premotor cortices, inferior parietal lobule, and primary motor cortex [1]-[5]. Whereas there is no doubt that motor areas are activated during action observation, it is still not clear whether these activations are either in part or entirely due to mirror neurons [6] [7]. Mirror neurons were first found in the macaque monkey F5 area in premotor cortex and discharge when an action of the same type is either executed or observed [8]-[10]. Subsequent studies confirmed homologous human activity in the rostral part of the inferior parietal lobule [Brodmann's area (BA) 40] and the lower part of the precentral gyrus (BA6, i.e., premotor area) plus the posterior part of the inferior frontal gyrus (BA44, i.e., Broca's area) [3] [4]. This network is known as the mirror neuron system (MNS) in human. However, the causal relationship between MNS and the other brain regions that have higher-order cognitive functions remain controversial [7] [11]-[14].

Recent studies indicate that some inhibitory mechanism is required to control automatic activity of MNS [15]-[17]. The activities in human MNS are modulated by the degree of intervention from other brain regions that have some higher-order cognitive functions, such as the cognitive control that inhibits unwanted behavior [15] [16]. For example, Cross et al. [16] investigated the interactions between the cognitive control and mirror neuron systems by using an "imitation-inhibition task" in which participants have to execute their index or middle finger movement, respectively, in response to the observed congruent (i.e., the same) or incongruent (i.e., the different) finger movement of a video-taped hand. In the incongruent trials, the stimulus and response were dissimilar. This conflict yielded more activation in the prefrontal neural network, specifically in the anterior insula associated with cognitive control system. Cross et al. [16] suggested that anterior insula suppresses the automatic activity in MNS in response to the observed incongruent actions.

Thus, some studies have reported that the activation of these higher-order cognitive functions leads to differences in activities of MNS when the stimuli are not ambiguous such as grasping actions. However, it is still controversial whether the activities in MNS more sensitively reflect degree of engagement of higher-order cognitive functions when stimuli are ambiguous such as incomplete human-like objects. The first aim of the present study was to investigate the influence of higher-order cognitive functions on the activities in the fronto-parietal mirror neuron circuit during perception of more abstract and ambiguous postures (i.e., simplified pseudo-postures) that elicit a variety of interpretations.

Although it is widely acknowledged that the MNS is involved in action perception, it is not clear whether activity within the MNS depends on a tight match between actor and observer in terms of their forms-human versus robot [18]. Some studies have reported that the MNS is activated by the observation of both human and robot actions, with no significant differences between these two agents [19]-[21], whereas others have argued that the MNS either does not respond or weakly responds when the perceived actor is robot compared with human [22]-[24]. The second aim of the present study was to examine whether the observation of sequentially presented still images of human-like objects elicits the activation of MNS or not.

Previous studies have shown that the fronto-parietal mirror neuron circuit responds not only to full displays of body actions, but also to point-light displays of human actions [25] and to static images implying a body action [26]-[28]. The premotor cortex is activated by the presentation of sequential events that represent human body postures not implying an action [29]-[32], or those that do not represent human figures (e.g., round) [33]. For example, Saito and his colleagues have reported that the inferior frontal mirror neuron area (the lower part of the precentral gyrus and the posterior part of inferior frontal gyrus) may be activated when observers infer a kinematic relation between sequentially presented artificial human postures depicted with three-dimensional computer graphics [29]. The kinematic relation is subject to the flexibility of joints of the human body. This result suggests that our perceptual system could function to perceive observed objects as human by means of conceptually driven processing [34] [35]. It is important to note that the still images of the human body postures are not purely linked by "apparent motion". In this study, the inter-stimulus interval (ISI) between the two postures was $1 \mathrm{~s}$, because the apparent motion studies suggest that viewing human posture becomes linked by apparent motion in less than $750 \mathrm{~ms}$ ISI [36]-[38].

However, it is still not clear how higher-order cognitive functions such as cognitive control or a tendency of retrieving the conceptual knowledge of human body posture or bodily movement affect activity in the MNS. The present study was designed to address this issue by using the posture recognition task similar to Ito et al. [29] and more abstract and ambiguous objects, i.e., simplified pseudo-postures that could have many individual interpretations rather than human postures. Figure 1 illustrates a human posture used in Ito et al. [29] and a pseudo-posture (i.e., wrists, head and legs removed) used in the present study. The participant's task was to observe a 


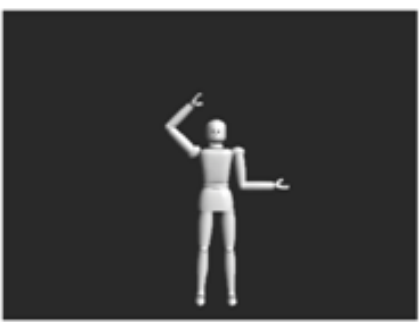

(a)

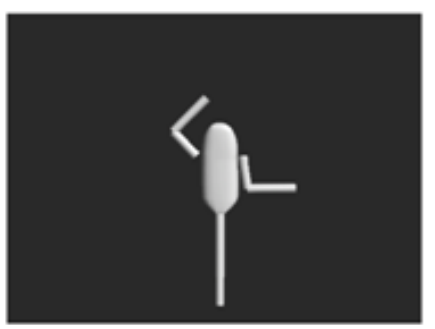

(b)

Figure 1. Sample stimuli. (a) Human posture used in Ito et al. [26]; (b) Pseudo-posture used in this study.

list of five asymmetrical face-to-face target pseudo-postures sequentially presented and to judge whether a test pseudo-posture was identical to one of these preceding target postures. In the posture recognition task, complexities of the kinematic relation between the neighboring pseudo-postures were manipulated as a function of the number of joints responsible for a postural change between the neighboring pseudo-postures. The stimulus lists consisted of five target pseudo-postures that varied in the number of joints (one vs. four), named 1-step and 4-step conditions. The 1-step condition produced a smaller number of postural changes between a preceding pseudo-posture and a subsequent pseudo-posture, while the 4-step condition produced a larger number of postural changes. In the 4-step condition, the four joints varied simultaneously between a preceding pseudo-posture and a subsequent pseudo-posture. If the five target pseudo-postures are processed independently, there should be no difference in the recognition performances and brain activities between the 1-step and 4-step conditions.

We used this posture recognition task in order to investigate the influence of higher-order cognitive functions on the activities in the fronto-parietal mirror neuron circuit during the perception of the pseudo-postures. In the present study, we measured brain activation during the posture recognition task using near-infrared spectroscopy (NIRS). Our main aim in the experiment was to investigate whether the activity in MNS is affected by the degree of the activation of higher-order cognitive functions that are involved in visual perception of human body postures and bodily movement. In order to determine this, we examined the activities in the frontal mirror neuron area (the lower part of the precentral gyrus and the posterior part of the inferior frotal gyrus) during the perception of sequentially presented pseudo-postures.

In this experiment, we also focused on the intrusion of verbal encoding, i.e., the activations in the inferior frontal mirror neuron area might be caused by subvocalization during the observation of pseudo-postures. In order to test the effects of subvocalization, the observation of pseudo-postures was contrasted with two types of novel contour shapes that were covertly easy versus difficult to name as a control condition. We predict that a relatively higher activation in the posterior part of the left superior temporal gyrus and the angular gyrus (agreeing with the location of Wernicke's area) may be observed due to the covert naming when the novel contour shapes are likely to be easily named [39] [40], while there will be little or no activation in the posterior part of the left inferior frontal gyrus (agreeing with the location of Broca's area) and the sensory-motor cortex. In contrast, during the observation of pseudo-postures, we predict that a relatively higher activation in Broca's area and the sensory-motor cortex may be observed, while there will be little or no activation in the auditory cortex.

We employed multichannel NIRS to measure the brain activity in the left frontal, left temporal, and occipital cortices. NIRS is a noninvasive neuroimaging method that measures cortical haemodynamic responses by using near-infrared light emitted from the emitter optodes placed on a person's head [41]-[43] (for a review see [44]). Although NIRS has limitations such as a relatively low spatial resolution, it has an advantage-few physical constraints on the participants-in comparison to fMRI, PET, and MEG. Specifically, it has been reported that participants in a restricted hand condition retrieved and subsequently recalled significantly less words or events than participants whose hands were unrestricted [45] [46]. Taking into account the relation between physical constraints and memory load, in this experiment we actively used NIRS that imposed relatively few physical constraints on the participants.

\section{Methods}

\subsection{Participants}

Fourteen healthy right-handed (under) graduate students (6 males and 8 females; age: 18 to 27 years) from Na- 
goya University (Nagoya, Japan) participated in the experiment for pay. Written informed consent was obtained from all participants. All participants were native Japanese and naive to the purpose of the study, which was approved by the local ethics committee of the department of cognitive informatics at Nagoya University.

\subsection{Materials and Design}

Endo, Saiki, Nakao, and Saito [47] designed the 100 novel contour shapes and clarified what geometrical properties contributed to complexity and similarity judgments of the novel contour shapes. We selected the top 30 shapes which are easy to name due to a high association value between a contour shape and an object, and the bottom 30 shapes which are difficult to name in the 100 novel contour shapes. Figure 2 illustrates examples of the novel contour shapes used in this study. The novel contour shapes were presented as white line-drawings on a black background.

Endo and his colleagues estimated the association values of the 60 novel contour shapes in their preliminary study [48]. In order to confirm the difference of association values between easy-to-name and difficult-to-name shapes, we conducted a one-way repeated-measures analysis of variance (ANOVA) with the picture-naming of novel contour shapes (easy-to-name vs. difficult-to-name). It showed a significant main effect of the picturenaming. This result indicated that the averaged association value in the easy-to-name condition (mean value $=$ 83.7) was higher than that in the difficult-to-name condition (mean value $=65.8)(F(1,58)=441.62, p<0.01)$. This suggests that participants would be more likely to subvocalize easy-to-name as compared to difficult-toname shapes.

The target lists used in this experiment consisted of five novel contour shapes that were selected on the basis of the combinations of the easy-to-name shapes or the combinations of the difficult-to-name shapes. These easyto-name and difficult-to-name shapes were not mixed within a target list. We designated the two picture-naming conditions for easy-to-name and difficult-to-name shapes as easy and difficult conditions, respectively.

The human posture used in Ito et al. [29] was replaced by five cylinder arms and one ellipsoid body. We produced 100 pseudo-postures based on the combinations of left/right shoulder orientations $\left(0^{\circ}, 45^{\circ}, 90^{\circ}, 135^{\circ}\right.$, and $180^{\circ}$ ) defined by clockwise or counterclockwise rotations from the upright position in the vertical plane, and left/right elbow orientations $\left(0^{\circ}\right.$ and $\left.90^{\circ}\right)$ defined in the same manner as the shoulder. We then excluded 32 pseudo-postures due to their high discriminability. That is, 19 pseudo-postures had the limbs twisted in a biomechanically impossible manner. Nine pseudo-postures were symmetrical with respect to the median line, and four pseudo-postures were symmetrical with respect to a point. We finally used 68 basic pseudo-postures. These pseudo-postures were designed using a three-dimensional computer graphics software (Shade R5; E-Frontier Inc., Tokyo, Japan). Figure 3 illustrates examples of the pseudo-postures used in this study.

The number of joints functioning during the changes between a preceding pseudo-posture and the subsequent pseudo-posture was calculated for any combination of 2 pseudo-postures from 68 basic pseudo-postures. The minimum number of joints was one, while the maximum number was four. We obtained 720 combinations of one joint, and 1024 combinations of four joints. The target lists consisted of five pseudo-postures that were selected from the combinations of one or four joints. We designated the joint conditions for one joint and four joints as the 1-step and 4-step conditions, respectively.

The novel contour shapes and pseudo-postures were displayed on a 17-inch monitor located at a distance of approximately $50 \mathrm{~cm}$. All stimuli fit within the area of $8.5 \times 7 \mathrm{~cm}$ on the monitor. None of the stimuli in a target list were used twice in the same target list.

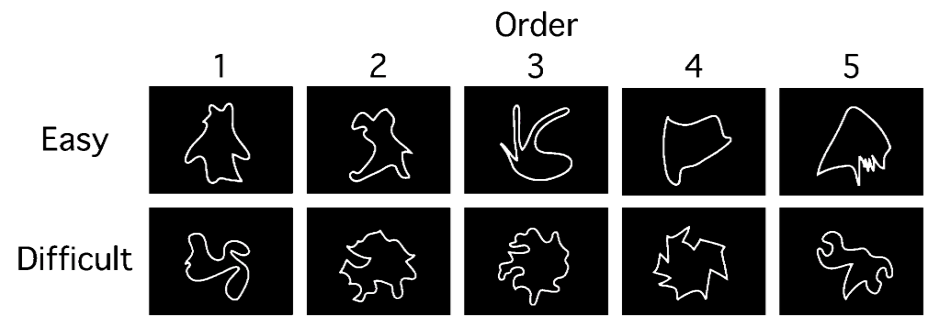

Figure 2. Examples of novel contour shapes. The novel contour shapes used in the easy and difficult conditions are shown in the top and bottom rows, respectively. 


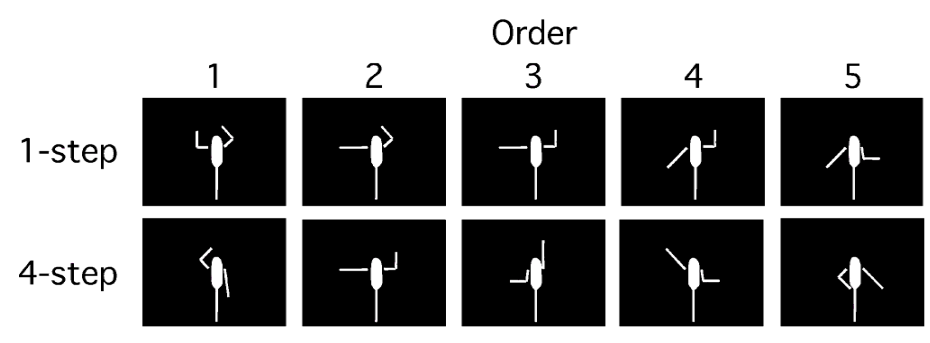

Figure 3. Examples of pseudo-postures. The pseudo-postures used in the 1 -step and 4-step conditions are shown in the top and bottom rows, respectively.

The participants were presented two kinds of visual stimuli, i.e., novel contour shapes and pseudo-postures. Each stimulus type was run in a separate block. We used the ease of picture-naming in the novel contour shape condition (i.e., easy and difficult) and the number of steps in the pseudo-posture condition (i.e., one and four steps) as the independent variables.

\subsection{Procedure}

The participants were tested individually. They were seated comfortably and asked to observe sequentially presented stimuli (novel contour shapes or pseudo-postures) and to judge whether a test stimulus was identical to one of the preceding five target stimuli. One experimental trial consisted of three experimental phases, i.e., study phase, test phase, and confidence judgment phase.

In the study phase, each trial was begun by presenting a white fixation point on a black background for $1 \mathrm{~s}$, followed by a blank interval of $1 \mathrm{~s}$ with the presentation of the first target stimulus. The first target stimulus was presented for $1 \mathrm{~s}$. A blank screen was displayed for $1 \mathrm{~s}$, followed by the second target stimulus presented for the same duration as the first. Thus, both the stimulus duration and the stimulus onset asynchrony were $1 \mathrm{~s}$. The fixation point and the target stimuli were displayed $8.5 \mathrm{~cm}$ to the left of the center of the screen. The participants were instructed to observe the sequentially presented stimuli and memorize them.

In the test phase, the fixation point was displayed for $1 \mathrm{~s}$, followed by a blank interval of $1 \mathrm{~s}$. Then, a test stimulus was presented until the participants responded. The fixation point and test stimulus were displayed $8.5 \mathrm{~cm}$ to the right from the center of the screen in order to eliminate the effect of the afterimage of the target stimuli on the screen. The task of the participants was to make a recognition judgment (yes or no) for the test stimulus. They were asked to press the "Y" key with the right index finger when the test stimulus was presented in the study phase, and to press the "N" key with the left index finger when it was not presented. They were encouraged to respond as quickly and accurately as possible. Participants were not given feedback on whether their response was correct or not.

In the confidence judgment phase, the participants were asked to rate the degree of their confidence in recognizing the answer being correct on a 4-point scale (from 1 = the least confident to $4=$ the most confident). This confidence judgment was used for motivation to attend to the recognition task. After responding, participants were given an inter-trial interval of $10 \mathrm{~s}$.

Prior to participating in the experimental trials, each participant completed a block of 12 practice trials. In the practice trials, the participants were asked to perform recognition after the presentation of digits from one to nine instead of novel contour shapes or pseudo-postures. The experimental session consisted of two blocks. Each stimulus type was run in a separate block. The two experimental blocks were fixed in an order of the novel contour shape and then pseudo-posture condition across participants. The first and second experimental blocks consisted of 12 trials in each block. The order of the easy and difficult conditions was counterbalanced across participants. In each picture-naming condition, half of the trials were assigned to the positive set in which a positive response was required, and the other half were assigned to the negative set in which a negative response was required. The positive/negative set was randomized within each picture-naming condition across participants. The order of the 1-step/4-step conditions and the number of positive/negative sets in the pseudo-posture condition were prepared in the same manner as those in the novel contour shape condition. After each experimental block there is a 2 minute rest period. The experimental session lasted for approximately 30 minutes.

After the recognition task, participants were given a questionnaire to report whether they had considered the 
pseudo-postures as the human postures or not. And then they were interviewed by one of the authors.

\subsection{NIRS Data Acquisition}

A 24-channel NIRS unit operated at 780 and 830 nm wavelengths (ETG-100; Hitachi Medical Co., Tokyo, Japan) was used to measure the temporal changes in oxygenated haemoglobin (oxy-Hb), deoxygenated haemoglobin (deoxy-Hb), and total haemoglobin (total-Hb) concentrations. Nine optodes were placed on the left temporal and occipital regions in a lattice pattern to form 12 channels for each region $(6 \times 6 \mathrm{~cm})$. The middle optode in the lowest line on the left side was located on the nearby T3 position (according to the international 10 - 20 system for electroencephalogram recording). The center column of the optodes on the occipital region was adjusted to align with the inion (Figure 4). Each channel consisted of 1 emitter optode and 1 detector optode located at a distance of $3 \mathrm{~cm}$ from the emitter optode. The sampling rate at each channel was $100 \mathrm{~ms}$.

We focused on the oxy- $\mathrm{Hb}$ concentration changes during the recognition task because oxy- $\mathrm{Hb}$ is the most sensitive parameter of regional cerebral blood flow [41] [49]. The average oxy-Hb concentration change for each channel was obtained for the measurement periods. The time course of concentration changes in oxy- $\mathrm{Hb}$ consisted of the following periods: pre-task (2 s), first-fixation (2 s), target-presentation (divided into 5 periods: $5 \times 2 \mathrm{~s}=10 \mathrm{~s}$ ), second-fixation ( $2 \mathrm{~s}$ ), test-presentation (approximately $1 \mathrm{~s}$ ), confidence-judgment (approximately $3 \mathrm{~s}$ ), and post-task (divided into 2 periods: $2 \times 4 \mathrm{~s}=8 \mathrm{~s}$ ). The raw data of oxy-Hb in response to the recognition task in each trial and on each channel were converted into $z$-scores that were calculated using the mean value and the standard deviation of oxy-Hb changes during the pre-task period [50] [51]. We finally obtained groupaveraged z-scores for each condition.

\section{Results}

\subsection{Behavioral Performance}

All statistical analysis was carried out by Statistical Packages for Social Sciences (SPSS 19; IBM, Tokyo, Japan). The overall average hit rate in the novel contour shape condition (easy: 86\%; difficult: $81 \%$ ) and that in the pseudo-posture condition (1-step: 76\%; 4-step: 83\%) were higher than the 50\% chance level. The proportions of hits were entered into a one-way ANOVA with the ease of picture-naming in the novel contour shape condition (easy and difficult) as the within-subject factor, and the number of steps in the pseudo-posture condition (1-step and 4-step) as the within-subject factor, independently. There were no significant main effects for the ease of picture-naming $(F<1)$ or the number of steps $(F(1,13)=1.27, n s)$.

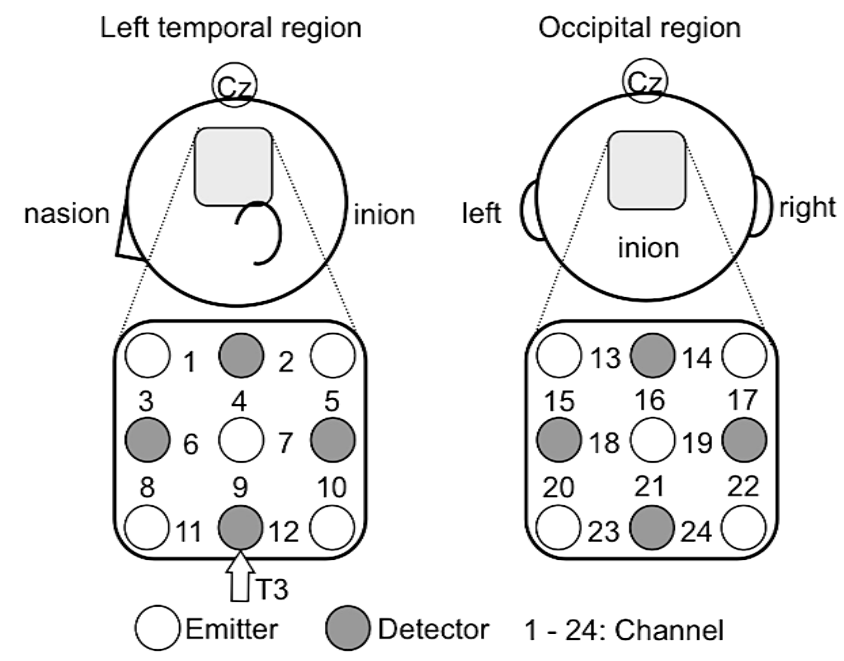

Figure 4. Location of the optodes placed on the left temporal and occipital regions. The distance between each emitter and the corresponding detector was set at $3 \mathrm{~cm}$. T3 was located in the region between channels 11 and 12 on the left temporal region. The center column of the optodes on the occipital region was adjusted to align with the inion. 
The overall average correct rejection rates in the novel contour shape condition (easy: 88\%; difficult: 95\%) and that in the pseudo-posture condition (1-step: 67\%; 4-step: 90\%) were higher than the 50\% chance level. The proportions of correct rejections were entered into a one-way ANOVA with the ease of picture-naming in the novel contour shape condition (easy and difficult) as the within-subject factor, and the number of steps in the pseudo-posture condition (1-step and 4-step) as the within-subject factor, independently. There was no significant main effect for the ease of picture-naming $(F(1,13)=1.01, n s)$. On the other hand, there was a significant main effect for the number of steps $(F(1,13)=6.19, p<0.05)$. This main effect of the number of steps was due to the high correct rejection rate under the 4-step condition as compared to that under the 1-step condition. These results in the step condition are consistent with the results in Ito et al. [29] showing that the target postures are not processed independently.

\subsection{NIRS Data in the Novel Contour Shape Condition}

The experimental trials associated with incorrect responses (i.e., miss or false alarm) were excluded from further analysis. We analyzed the experimental trials associated with correct responses (i.e., hit or correct rejection). We analyzed the group-averaged values of the z-scores for oxy-Hb concentration changes during each period except the pre-task period, that is, from the first-fixation period to the second-post-task period (the 11 measurement periods). To compare the brain activity in the easy and difficult conditions, we conducted a two-way repeatedmeasures ANOVA [2 (easy and difficult) $\times 11$ (measurement periods)] of the $z$-scores for each channel. To avoid the effect caused by multiple comparisons, an $\alpha$ level of $<0.05$ after Shaffer's modified sequentially rejective Bonferroni correction for multiple comparisons (hereafter, Shaffer's modified Bonferroni correction) was used to identify significant associations. We focused on the channels that showed a significant main effect of the ease of picture-naming, and an interaction between the picture-naming condition and the period because we presumed that the cortical haemodynamic responses would be modulated by the ease of picture-naming.

In the left temporal region, we found significant main effects of the ease of picture-naming in channels 5 , 7 , and $10(F(1,13)=14.44, p<0.01 ; F(1,13)=6.40, p<0.05 ; F(1,13)=8.92, p<0.05$, respectively $)$, and significant interactions between the picture-naming condition and period in channels 1 and $9(F(10,130)=2.05, p<$ $0.05 ; F(10,130)=2.39, p<0.05$, respectively). Post-hoc comparisons using Shaffer's modified Bonferroni correction revealed that these interactions were due to the large responses under the easy condition as compared to those under the difficult condition during the target-presentation period $(p<0.05)$.

On the other hand, in the occipital region, we found significant main effects of the ease of picture-naming in channels 13, 15, 20, 21, and $24(F(1,13)=6.00, p<0.05 ; F(1,13)=6.37, p<0.05 ; F(1,13)=5.99, p<0.05$; $F(1,13)=4.87, p<0.05 ; F(1,13)=9.63, p<0.01$, respectively), and significant interactions between the picture-naming condition and period in channels 13, 15, 16, 20, 22, 23, and $24(F(10,130)=2.09, p<0.05$; $F(10$, $130)=2.25, p<0.05 ; F(10,130)=2.34, p<0.05 ; F(10,130)=2.00, p<0.05 ; F(10,130)=2.07, p<0.05$; $F(10,130)=3.00, p<0.01 ; F(10,130)=3.46, p<0.01$, respectively). Post-hoc comparisons using Shaffer's modified Bonferroni correction revealed that these interactions were due to the large responses under the easy condition as compared to those under the difficult condition during the period from target-presentation to confidence-judgment $(p<0.05)$. Taken together, in all the channels that showed significant differences between the easy and difficult conditions, the oxy-Hb concentrations under the easy condition were higher than those under the difficult condition. We found that higher activation occurs in the left temporal region (channels 5, 7, 9, and 10) and the occipital region (channels 13, 15, 16, 20, 21, 22, 23, and 24) during the observation of novel contour shapes in the easy condition than in the difficult condition. Figure 5 shows the average time courses of the changes in the oxy-Hb concentrations in three representative channels (for the entire results, see Appendix A).

Based on the three-dimensional probabilistic anatomical craniocerebral correlation [51], T3 was projected onto the middle temporal gyrus. We presumed that the left channels 5, 7, 9, and 10 roughly cover the posterior part of the left superior temporal gyrus and the angular gyrus. The above results suggest that the left auditory cortex (the channels 9 and 10) and Wernicke's area (the channels 5 and 7) are activated during the observation of easy-to-name shapes. Due to the relatively low spatial resolution of the NIRS measurement, we could not determine precisely whether the posterior part of the left superior temporal gyrus and the angular gyrus are the only regions involved in naming of novel contour shapes. However, considering that the auditory cortex and Wernicke's area are involved, our results lead to a speculation that the activations in these regions during the observation of easy-to-name shapes might be related to the production of auditory imagery [39] [40] and phonological 

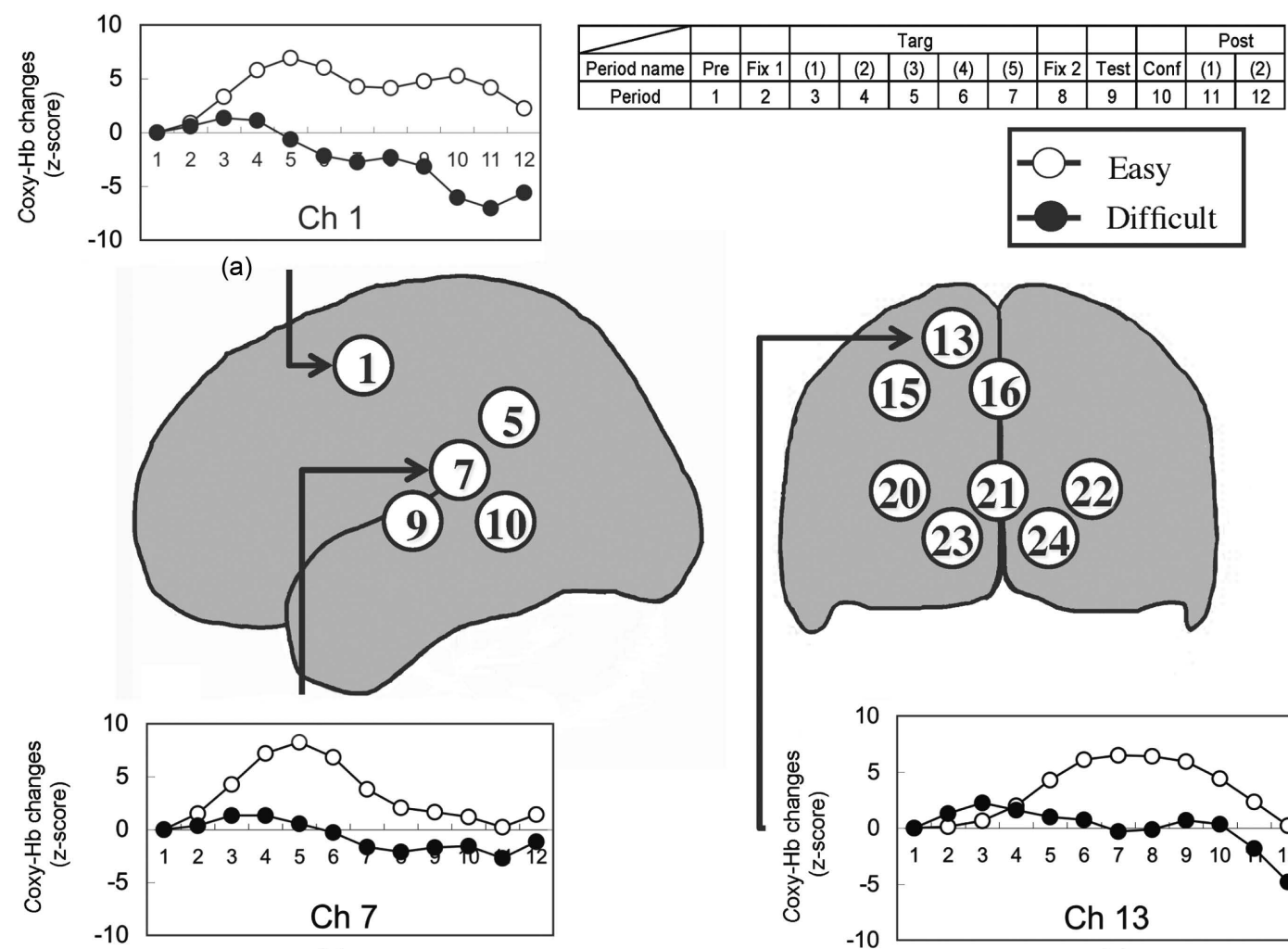

(b)

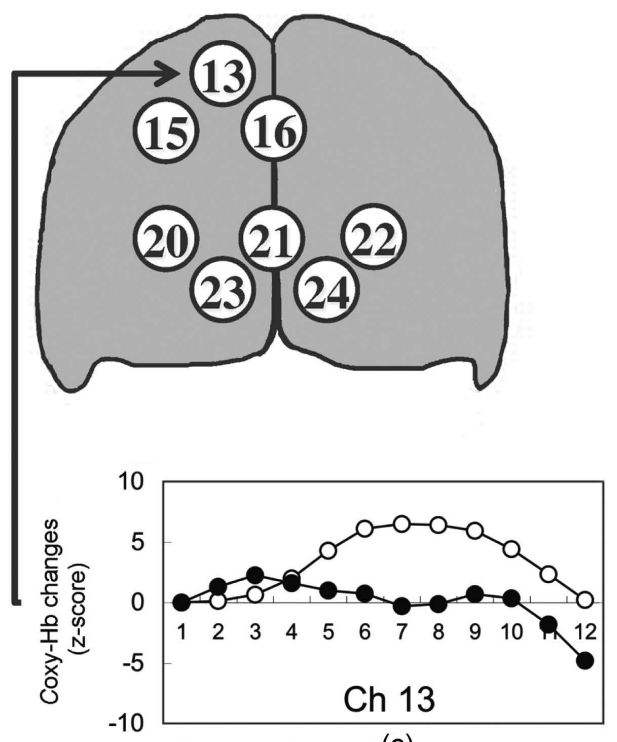

(c)

Figure 5. Average oxy-Hb concentration changes under the novel contour shape condition during the correct responses. Three representative channels are presented for each picture-naming condition. (a) Channel 1; (b) Channel 7; (c) Channel 13. The numbers inside a circle represent the measurement channels. Abbreviations: Pre, pre-task; Fix 1, first-fixation; Targ, target-presentation; Fix 2, second-fixation; Test, test-presentation; Conf, confidence-judgment; Post, post-task.

encoding [52] [53].

We presumed that the channels $13,15,16,20,21,22,23$, and 24 roughly cover the occipital lobe. The results in the novel contour shape condition showed that the occipital visual cortex is activated during the observation of easy-to-name shapes. We can now interpret the activation of the occipital cortex as due to the visual processing related to the picture-naming in which a visual representation of the object is computed from the visual image.

\subsection{NIRS Data in the Pseudo-Posture Condition}

To compare the brain activity in the 1-step and 4-step conditions, we conducted a two-way repeated-measures ANOVA [2 (1-step and 4-step) $\times 11$ (measurement periods)] of the $z$-scores on each channel. We focused on the channels that showed a significant main effect of the step number and interaction between the step condition and the period because we presumed that the cortical haemodynamic responses would be modulated by the number of steps.

In the left temporal region, we found significant main effects of the step number in channel $1(F(1,13)=6.49$, $p<0.05)$, and interactions between the step condition and period in channels 6 and $8(F(10,130)=2.16, p<$ $0.05 ; F(10,130)=2.87, p<0.01$, respectively). On the other hand, there were no significant main effects of the step number and interactions in the occipital region. Post-hoc comparisons using Shaffer's modified Bonferroni correction revealed that these interactions were due to the large responses under the 4-step condition as compared to those under the 1-step condition during the post-task periods both in channel 6 and $8(p<0.05)$, while the interaction in channel 6 was also due to the large response under the 1-step condition as compared to that under the 4 -step condition during the target-presentation period $(p<0.05)$. Focusing on the brain activity during 
the target presentation period, higher activation occurred under the 1-step condition in channel 6, but not in channel 8. The activation under the 1-step and 4-step conditions in channel 8 showed a comparable level. These results suggest that the brain activity in the left frontal region is modulated by the number of joints, and the brain function in channel 6 is distinctly different from that in channel 8.

Figure 6 shows the average time courses of the changes in the oxy-Hb concentrations in channels 6 and 8 . Based on the three-dimensional probabilistic anatomical craniocerebral correlation [54], we presumed that the left channels 6 and 8 roughly cover the lower part of the precentral gyrus (BA6) and the posterior part of the inferior frontal gyrus (BA44), respectively. The data of present study, showing the different patterns of activation in the channels 6 and 8, is consistent with the previous studies that have reported the distinct functions in the inferior frontal mirror neuron area [55]-[60]. Thus, we carried out further analysis focused on channels 6 and 8.

Referring to the introspection sheets of 14 participants, they were divided into two groups according to whether they perceived the pseudo-postures as the human postures or not: 10 of them marked that they perceived the pseudo-postures as the human postures (High Sensitivity group, HS), while four of them did not (Low Sensitivity group, LS). Figure 7 shows the activation patterns in channels 6 and 8 for each group. The HS group demonstrated the same pattern of activation in channels 6 and 8 as did the 14-participants group. In the HS group, we found significant interactions between the step condition and period in channels 6 and $8(F(10,90)=$ $2.75, p<0.05 ; F(10,90)=2.18, p<0.05$, respectively). Post-hoc comparisons using Shaffer's modified Bonferroni correction revealed that the interaction in channel 6 was due to the large response under the 1-step condition as compared to that under the 4-step condition during the target-presentation period, and also due to the large responses under the 4-step condition as compared to those under the 1-step condition during the post-task periods $(p<0.05$ ), while the interaction in channel 8 was only due to the large responses under the 4-step condition as compared to those under the 1-step condition during the post-task periods $(p<0.05)$. These results in the HS group accord with the results of the 14 participants.

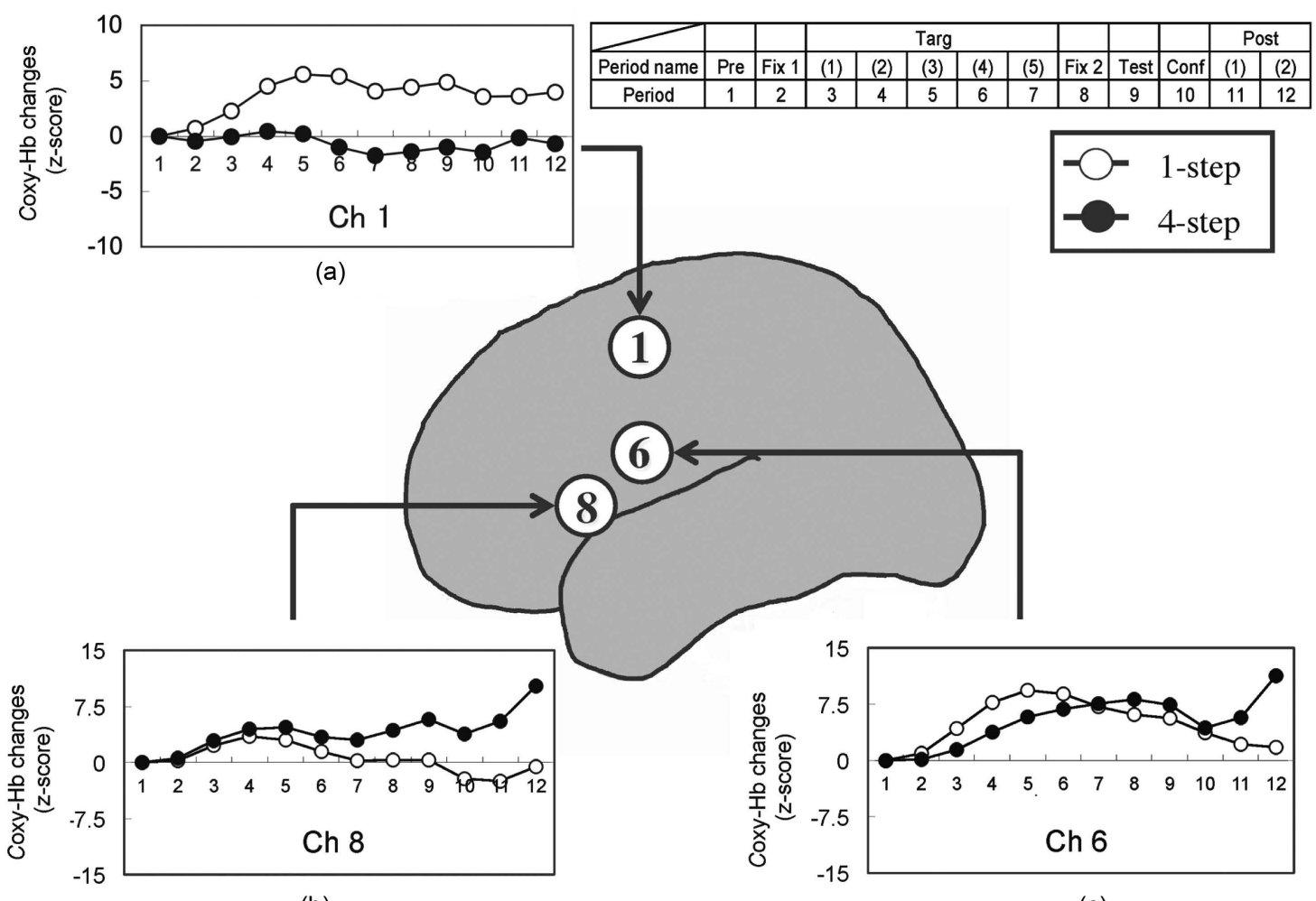

(b)

(c)

Figure 6. Average oxy-Hb concentration changes under the pseudo-posture condition during the correct responses. The changes in channels 1(a), 8(b), and 6(c) are presented for each step condition. Abbreviations: Pre, pre-task; Fix 1, first-fixation; Targ, target-presentation; Fix 2, second-fixation; Test, test-presentation; Conf, confidence-judgment; Post, post-task. 
Ch 8

14-participants
group
$(n=14)$

\section{Low Sensitivity group $(n=4)$}

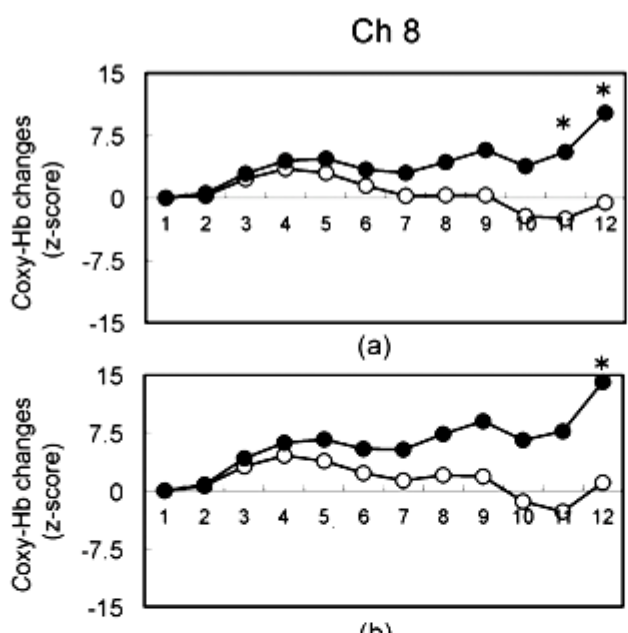

(b)

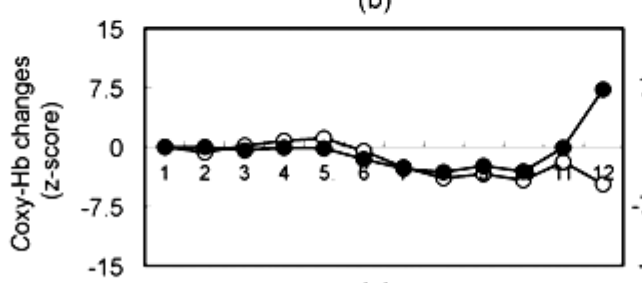

(c)

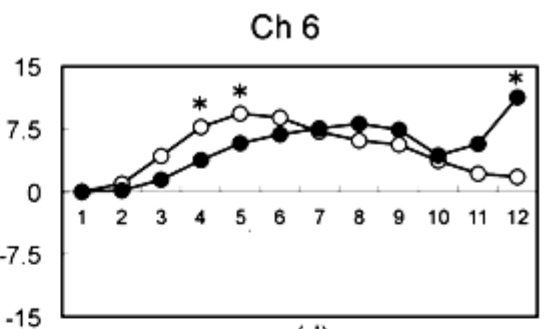

(d)

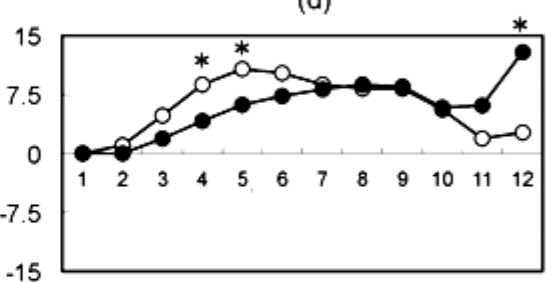

(e)

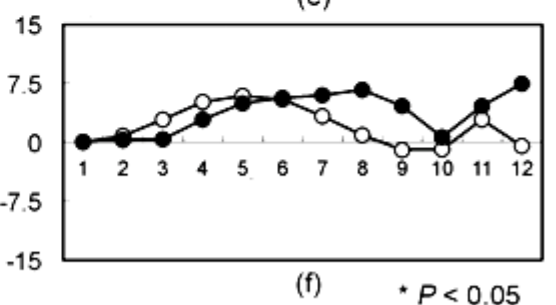

(f) $\quad * P<0.05$

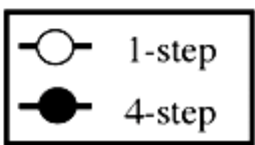

Figure 7. Average oxy-Hb concentration changes under the pseudo-posture condition during the correct responses for each group. (a) Channel 8 in the 14-participants group; (b) Channel 8 in the HS group; (c) Channel 8 in the LS group; (d) Channel 6 in the 14-participants group; (e) Channel 6 in the HS group; (f) Channel 6 in the LS group. The changes in channels 6 and 8 are presented for each step condition. Abbreviations: Pre, pre-task; Fix 1, first-fixation; Targ, target-presentation; Fix 2, second-fixation; Test, test-presentation; Conf, confidence-judgment; Post, post-task.

The LS group did not show the same pattern of activation with the HS group in channel 8, while it showed the same pattern of activation in channel 6. We did not find significant main effects and interactions in channels 6 and 8 in the LS group because of the small sample size lacking of statistical power within the LS group. Although the relatively small sample size in the current study means that our results should be interpreted with caution, in the LS group, both the 1-step and 4-step conditions in the channel 8 seemed to show activation on the level of the base line during the target-presentation period, which is dissimilar to the activation patter in the channel 8 in the HS group. Furthermore, the channel 6 in the LS group seemed to show relatively higher activation to the base line, which is similar to the activation pattern in channel 6 in the HS group.

These results in the HS group and the LS group might suggest that the perception of pseudo-postures as human postures, or the extraction of human-likeness from the sequentially presented pseudo-postures triggers the posterior part of the inferior frontal gyrus (BA44: channel 8) to visualize the kinematic relation between sequentially presented peudo-postures as a human bodily movement.

\subsection{NIRS Data both in the Novel Contour Shape and Pseudo-Posture Conditions}

We found a significant activation in the left frontal region (channel 1) both in the novel contour shape and the pseudo-posture conditions. These results indicated that the average values of the $\mathrm{z}$-scores for oxy-Hb concentration changes under the easy condition were higher than those under the difficult condition, while the group-averaged values of the $z$-scores for oxy-Hb concentration changes under the 1-step condition were higher than those under the 4-step condition.

Based on the 3-dimensional probabilistic anatomical craniocerebral correlation [54], we presumed that the left 
channel 1 roughly covers the posterior frontal cortex, which is consistent with the dorsolateral premotor cortex (dPMC). Some studies indicated that the dPMC retains cognitive functions (e.g., spatial and nonspatial working memory or mental operation) as well as motor functions [61] [62]. Thus, the higher activity in that area may be related to such cognitive load in the recognition memory task. Future studies are required for investigating the functional relationship between the APMC and the lower part of the precentral gyrus for the visual perception of human-like body postures.

\section{Discussion}

The purpose of the present study was to investigate how higher-order cognitive functions affect the activities in the fronto-parietal mirror neuron circuit during the perception of simplified pseudo-postures. In order to address this issue, we examined brain activity during the observation of sequentially presented pseudo-postures.

First, in this study, we compared the brain activation during the observation of pseudo-postures and novel contour shapes. The present data for the pseudo-postures demonstrated that distinct activation patterns are observed in the frontal mirror neuron circuit consisting of the two cortical areas, while no activation related to the number of steps is observed in the left superior temporal gyrus, left angular gyrus, or occipital cortex (i.e., the areas related to the verbal encoding). This seems to imply that the MNS may respond to artificial human-like objects [19]-[21]. In contrast, the data for the novel contour shapes demonstrated that the activation is observed in the areas related to the verbal encoding, while no activation related to the ease of picture-naming is observed in the frontal mirror neuron circuit.

Second, we verified that higher activation occurred under the 1-step condition in the left channel 6, but not in the left channel 8 during the target presentation period, showing a different pattern of activation in the lower part of the precentral gyrus (BA6) and the posterior part of the inferior frontal gyrus (BA44), respectively. In order to assess the effects of the higher-order cognitive functions, i.e., retrieving the conceptual knowledge of human body posture for perceiving the pseudo-postures as human postures and inhibitory control of automatic response tendencies evoked by human-like stimuli, we divided the 14 participants into a high sensitivity group (10 participants) who perceived the pseudo-postures as the human postures and a low sensitivity group (four participants) who did not. The HS group demonstrated the pattern of activation in the left channels 6 and 8 as did the 14-participants group, while the LS group did not. In particular, it is worthy to note that the LS group showed little or no activation in the left channel 8 (the posterior part of the inferior frontal gyrus; BA44), while the LS group showed a similar activation pattern with the HS group in the left channel 6, even though it was not significant in the LS group. Thus, observers who do not detect the possibility of human body form during the observation of individual pseudo-postures seem to show no activation of the BA44. This could be explained by the involvement of BA44 in mapping of an externally triggered representation of an observed pseudo-posture onto internally retrieved representations of human body postures by identifying the similarity between the particular spatial configuration of limbs of the human body and that of the pseudo-posture. Therefore, this mapping process would be suppressed in the LS group.

If the lower part of the precentral gyrus (BA6) is modulated by the number of joints functioning in a similar structure to the human upper limbs in all participants regardless of the perception of a pseudo-posture as a human posture, presumably, the lower part of the precentral gyrus is involved in computing configurational changes of sequentially presented pseudo-postures. The present data in the lower part of the precentral gyrus is consistent with previous studies that have shown the involvement of the premotor area in the representation of sequential information such as the motor plans [58]-[60].

Whereas the lower part of the precentral gyrus (BA6) is modulated by the number of joints responsible for a postural change between the neighboring postures, the posterior part of the inferior frontal gyrus (BA44) is modulated by the perception of a pseudo-posture as a human posture. Thus, the posterior part of the inferior frontal gyrus seems to be activated when participants attended to the biological characters (humanlike appearance) of the pseudo-postures, but might be normally under inhibitory control. One of the most promising brain regions, which pertain to the inhibitory control of motoric embodiment, is the prefrontal cortex, i.e., the anterior part of inferior frontal gyrus [10] [16] and/or anterior insula [19]. This prefrontal control network could suppress the MNS activity in response to the degree of the configural similarity between observed objects and human body postures stored as conceptual knowledge. This inhibitory control account predicts that activation of human MNS arises first, quickly and automatically in response to not only a human body form but also an incomplete 
human-like object, but subsequently an inhibitory mechanism has to come into play, which allows us to detect distinctions between human and other object representations. If the brain regions related to cognitive control mechanism affect the activity of human MNS, further study will be needed to examine causal interactions between these regions and MNS and the time courses of the interactions. In terms of limitations, the relatively small sample size in the current study means that our results should be interpreted cautiously. Further study is also needed to examine the influence of higher-order cognitive functions on the activities in MNS using a large group of participants.

\section{Conclusion}

We conclude that the degree of the activation of higher-order cognitive functions leads to the distinctive activities in the inferior frontal mirror neuron area consisting of the lower part of the precentral gyrus and the posterior part of the inferior frontal gyrus. This implies that the activities in MNS are modulated by the degree of intervention from other brain regions that would be engaged in higher-order cognitive functions such as inhibitory and/or facilitative processing of human body or bodily movement.

\section{Acknowledgements}

We thank Paul Langman, PhD, for his careful reading of the manuscript and assistance with English usage.

\section{References}

[1] Iacoboni, M. (2009) Imitation, Empathy, and Mirror Neurons. Annual Review of Psychology, 60, 653-670. http://dx.doi.org/10.1146/annurev.psych.60.110707.163604

[2] Iacoboni, M., Woods, R.P., Brass, M., Bekkering, H., Mazziotta, J.C. and Rizzolatti, G. (1999) Cortical Mechanisms of Human Imitation. Science, 286, 2526-2528. http://dx.doi.org/10.1126/science.286.5449.2526

[3] Rizzolatti, G. and Craighero, L. (2004) The Mirror-Neuron System. Annual Reviews of Neuroscience, 27, $169-192$. http://dx.doi.org/10.1146/annurev.neuro.27.070203.144230

[4] Rizzolatti, G., Fogassi, L. and Gallese, V. (2001) Neurophysiological Mechanisms Underlying the Understanding and Imitation of Action. Nature Reviews Neuroscience, 2, 661-670. http://dx.doi.org/10.1038/35090060

[5] Rizzolatti, G. and Sinigaglia, C. (2010) The Functional Role of the Parieto-Frontal Mirror Circuit: Interpretations and Misinterpretations. Nature Reviews Neuroscience, 11, 264-274. http://dx.doi.org/10.1038/nrn2805

[6] Oosterhof, N.N., Tipper, S.P. and Downing, P.E. (2013) Crossmodal and Action-Specific: Neuroimaging the Human Mirror Neuron System. Trends in Cognitive Sciences, 17, 311-318. http://dx.doi.org/10.1016/j.tics.2013.04.012

[7] Oztop, E., Kawato, M. and Arbib, A.M. (2013) Mirror Neurons: Functions, Mechanisms and Models. Neuroscience Letters, 540, 43-55. http://dx.doi.org/10.1016/j.neulet.2012.10.005

[8] di Pellegrino, G., Fadiga, L., Fogassi, L., Gallese, V. and Rizzolatti, G. (1992) Understanding Motor Events: A Neurophysiological Study. Experimental Brain Research, 91, 176-180. http://dx.doi.org/10.1007/BF00230027

[9] Gallese, V., Fadiga, L., Fogassi, L. and Rizzolatti, G. (1996) Action Recognition in the Premotor Cortex. Brain, 119, 593-609. http://dx.doi.org/10.1093/brain/119.2.593

[10] Rizzolatti, G., Fadiga, L., Gallese, V. and Fogassi, L. (1996) Premotor Cortex and the Recognition of Motor Actions. Cognitive Brain Research, 3, 131-141. http://dx.doi.org/10.1016/0926-6410(95)00038-0

[11] Avenanti, A., Candidi, M. and Urgesi, C. (2013) Vicarious Motor Activation during Action Perception: Beyond Correlational Evidence. Frontiers in Human Neuroscience, 7, 1-8. http://dx.doi.org/10.3389/fnhum.2013.00185

[12] Hickok, G. (2013) Do Mirror Neurons Subserve Action Understanding? Neuroscience Letters, 540, 56-58. http://dx.doi.org/10.1016/j.neulet.2012.11.001

[13] Kilner, J.M. (2011) More than One Pathway to Action Understanding. Trends in Cognitive Sciences, 15, 352-357. http://dx.doi.org/10.1016/j.tics.2011.06.005

[14] Keysers, C. (2009) Mirror Neurons. Current Biology, 19, R971-R973. http://dx.doi.org/10.1016/j.cub.2009.08.026

[15] Brass, M., Ruby, P. and Spengler, S. (2009) Inhibition of Imitative Behavior and Social Cognition. Philosophical Transactions of the Royal Society B: Biological Sciences, 364, 2359-2367. http://dx.doi.org/10.1098/rstb.2009.0066

[16] Cross, K.A., Torrisi, S., Losin, E.A.R. and Iacoboni, M. (2013) Controlling Automatic Imitative Tendencies: Interactions between Mirror Neuron and Cognitive Control Systems. NeuroImage, 83, 493-504.

http://dx.doi.org/10.1016/j.neuroimage.2013.06.060 
[17] Cross, K.A. and Iacoboni, M. (2014) To Imitate or Not: Avoiding Imitation Involves Preparatory Inhibition of Motor Resonance. NeuroImage, 91, 228-236. http://dx.doi.org/10.1016/j.neuroimage.2014.01.027

[18] Saygin, A.P., Chaminade, T., Ishiguro, H., Driver, J. and Frith, C. (2012) The Thing That Should Not Be: Predictive Coding and the Uncanny Valley in Perceiving Human and Humanoid Robot Actions. Social Cognitive and Affective Neuroscience, 7, 413-422. http://dx.doi.org/10.1093/scan/nsr025

[19] Cross, E.S., Liepelt, R., Hamilton, A.F.C., Parkinson, J., Ramsey, R., Stadler, W. and Prinz, W. (2012) Robotic Movement Preferentially Engages the Action Observation Network. Human Brain Mapping, 33, 2238-2254. http://dx.doi.org/10.1002/hbm.21361

[20] Gazzola, V., Rizzolatti, G., Wicker, B. and Keysers, C. (2007) The Anthropomorphic Brain: The Mirror Neuron System Responds to Human and Robotic Actions. NeuroImage, 35, 1674-1684. http://dx.doi.org/10.1016/j.neuroimage.2007.02.003

[21] Oberman, M.L., McCleery, P.J., Ramachandran, S.V. and Pineda, A.J. (2007) EEG Evidence for Mirror Neuron Activity during the Observation of Human and Robot Actions: Toward an Analysis of the Human Qualities of Interactive Robots. Neurocomputing, 70, 2194-2203. http://dx.doi.org/10.1016/j.neucom.2006.02.024

[22] Kilner, J.M., Paulignan, Y. and Blakemore, S.J. (2003) An Interference Effect of Observed Biological Movement on Action. Current Biology, 13, 522-525. http://dx.doi.org/10.1016/S0960-9822(03)00165-9

[23] Press, C., Gillmeister, H. and Heyes, C. (2007) Sensorimotor Experience Enhances Automatic Imitation of Robotic Action. Proceedings of the Royal Society of London: B, 274, 2509-2514. http://dx.doi.org/10.1098/rspb.2007.0774

[24] Tai, F.Y., Scherfler, C., Brooks, J.D., Sawamoto, N. and Castiello, U. (2004) The Human Premotor Cortex Is “Mirror” Only for Biological Actions. Current Biology, 14, 117-120. http://dx.doi.org/10.1016/j.cub.2004.01.005

[25] Saygin, A.P., Wilson, S.M., Hagler Jr., J.H., Bates, E. and Sereno, M. (2004) Point-Light Biological Motion Perception Activates Human Premotor Cortex. Journal of Neuroscience, 24, 6181-6188. http://dx.doi.org/10.1523/JNEUROSCI.0504-04.2004

[26] Johnson-Frey, S.H., Maloof, F.R., Newman-Norlund, R., Farrer, C., Inati, S. and Scott, G.T. (2003) Action or HandObject Interactions? Human Interior Frontal Cortex and Action Observation. Neuron, 39, 1053-1058. http://dx.doi.org/10.1016/S0896-6273(03)00524-5

[27] Urgesi, C., Moro, V., Candidi, M. and Aglioti, S.M. (2006) Mapping Implied Body Actions in the Human Motor System. The Journal of Neuroscience, 26, 7942-7949. http://dx.doi.org/10.1523/JNEUROSCI.1289-06.2006

[28] Urgesi, C., Maieron, M., Avenanti, A., Tidoni, E., Fabbro, F. and Aglioti, S.M. (2010) Simulating the Future of Actions in the Human Corticospinal System. Cerebral Cortex, 20, 2511-2521. http://dx.doi.org/10.1093/cercor/bhp292

[29] Ito, H., Saito, H., Shiraishi, T., Shimizu, H. and Yamamoto, Y. (2008) Brain Responses to Meaningless Postures as Measured by Near-Infrared Spectroscopy. NeuroReport, 19, 1411-1415. http://dx.doi.org/10.1097/WNR.0b013e32830dd61c

[30] Ito, H., Saito, H., Shiraishi, T. and Yamamoto, Y. (2007) Factors Underlying the Binding of the Sequentially Presented Postures. Cognitive Studies: Bulletin of the Japanese Cognitive Science Society, 14, 425-436.

[31] Saito, H. and Shiraishi, T. (2002) Koui no setumeiworikainitsunaguchishiki-shori (The Knowledge Processing to Link the Explanation of an Action and the Understanding of It). In: Saito, H. and Kita, S., Eds., Gesture, Action, and Meaning, Kyoritsu Shuppan, Tokyo, 210-247.

[32] Shiraishi, T. and Saito, H. (2002) Koui no rikaiwosuikounitsunaguchishiki-shori (The Knowledge Processing to Link the Understanding of an Action and the Execution of It). In: Saito, H. and Kita, S., Eds., Gesture, Action, and Meaning, Kyoritsu Shuppan, Tokyo, 248-278.

[33] Schubotz, R.I. and von Cramon, D.Y. (2004) Sequences of Abstract Nonbiological Stimuli Share Ventral Premotor Cortex with Action Observation and Imagery. The Journal of Neuroscience, 24, 5467-5474. http://dx.doi.org/10.1523/JNEUROSCI.1169-04.2004

[34] Blake, R. and Shiffrar, M. (2007) Perception of Human Motion. Annual Review of Psychology, 58, 47-73. http://dx.doi.org/10.1146/annurev.psych.57.102904.190152

[35] Norman, D.A. and Rumelhart, D. (1975) Exploration in Cognition. Freeman, San Francisco.

[36] Kourtzi, Z. and Shiffrar, M. (1999) Dynamic Representations of Human Body Movement. Perception, 28, 49-62. http://dx.doi.org/10.1068/p2870

[37] Shiffrar, M. and Freyd, J.J. (1990) Apparent Motion of the Human Body. Psychological Science, 1, 257-264. http://dx.doi.org/10.1111/j.1467-9280.1990.tb00210.x

[38] Stevens, J.A., Fonlupt, P., Shiffrar, M. and Decety, J. (2000) New Aspects of Motion Perception: Selective Neural Encoding of Apparent Human Movements. NeuroReport, 11, 109-115. http://dx.doi.org/10.1097/00001756-200001170-00022 
[39] Kraemer, D.J.M., Macrae, C.N., Green, A.E. and Kelley, W.M. (2005) Musical Imagery: Sound of Silence Activates Auditory Cortex. Nature, 434, 158. http://dx.doi.org/10.1038/434158a

[40] Hunter, M.D., Eickhoff, S.B., Miller, T.W.R., Farrow, T.F.D., Wilkinson, I.D. and Woodruff, P.W.R. (2006) Neural Activity in Speech-Sensitive Auditory Cortex during Silence. Proceedings of the National Academy of Sciences of the United States of America, 103, 189-194. http://dx.doi.org/10.1073/pnas.0506268103

[41] Hoshi, Y., Kobayashi, N. and Tamura, M. (2001) Interpretation of Near-Infrared Spectroscopy Signals: A Study with a Newly Developed Perfused Rat Brain Model. Journal of Applied Physiology, 90, 1657-1622.

[42] Villringer, A. and Chance, B. (1997) Non-Invasive Optical Spectroscopy and Imaging of Human Brain Function. Trends in Neuroscience, 20, 435-442. http://dx.doi.org/10.1016/S0166-2236(97)01132-6

[43] Yamashita, Y., Maki, A. and Koizumi, H. (1996) Near-Infrared Topographic Measurement System: Imaging of Absorbers Localized in a Scattering Medium. Reviews of Scientific Instruments, 67, 730-732. http://dx.doi.org/10.1063/1.1146849

[44] Wolf, M., Ferrari, M. and Quaresima, V. (2007) Progress of Near-Infrared Spectroscopy and Topography for Brain and Muscle Clinical Applications. Journal of Biomedical Optics, 12, Article ID: 062104. http://dx.doi.org/10.1117/1.2804899

[45] Frick-Horbury, D. and Guttentag, R.E. (1998) The Effects of Restricting Hand Gesture Production on Lexical Retrieval and Free Recall. American Journal of Psychology, 111, 43-62. http://dx.doi.org/10.2307/1423536

[46] Stevanonim, E. and Salmon, K. (2005) Giving Memory a Hand: Instructing Children to Gesture Enhances Their Event Recall. Journal of Nonverbal Behavior, 29, 217-223. http://dx.doi.org/10.1007/s10919-005-7721-y

[47] Endo, N., Saiki, J., Nakao, Y. and Saito, H. (2003) Perceptual Judgments of Novel Contour Shapes and Hierarchical Descriptions of Geometrical Properties. The Japanese Journal of Psychology, 74, 346-353. http://dx.doi.org/10.4992/jjpsy.74.346

[48] Nakao, Y., Saiki, J. and Endo, N. (2000) Association Values of the Novel Contour Shapes. Unpublished Raw Data.

[49] Strangman, G., Culver, J.P., Thompson, J.H. and Boas, D.A. (2002) A Quantitative Comparison of Simultaneous BOLD fMRI and NIRS Recordings during Functional Brain Activation. NeuroImage, 17, 719-731. http://dx.doi.org/10.1006/nimg.2002.1227

[50] Matsuda, G. and Hiraki, K. (2006) Sustained Decrease in Oxygenated Hemoglobin during Video Games in the Dorsal Prefrontal Cortex: A NIRS Study of Children. NeuroImage, 29, 706-711. http://dx.doi.org/10.1016/j.neuroimage.2005.08.019

[51] Sato, Y., Sogabe, Y. and Mazuka, R. (2007) Brain Responses in the Processing of Lexical Pitch-Accent by Japanese Speakers. NeuroReport, 18, 2001-2004. http://dx.doi.org/10.1097/WNR.0b013e3282f262de

[52] Levelt, W.J.M., Praamstra, P., Meyer, A.S., Helenius, P. and Salmelin, R. (1998) An MEG Study of Picture Naming. Journal of Cognitive Neuroscience, 10, 553-567. http://dx.doi.org/10.1162/089892998562960

[53] Salmelin, R., Hari, R., Lounasmaa, O.V. and Sams, M. (1994) Dynamics of Brain Activation during Picture Naming. Nature, 368, 463-465. http://dx.doi.org/10.1038/368463a0

[54] Okamoto, M., Dan, H., Sakamoto, K., Takeo, K., Shimizu, K., Kohno, S., et al. (2004) Three-Dimensional Probabilistic Anatomical Cranio-Cerebral Correlation via the International 10-20 System Oriented for Transcranial Functional Brain Mapping. NeuroImage, 21, 99-111. http://dx.doi.org/10.1016/j.neuroimage.2003.08.026

[55] de Lange, F.P., Spronk, M., Willems, R.M., Toni, I. and Bekkering, H. (2008) Complementary Systems for Understanding Action Intentions. Current Biology, 18, 454-457. http://dx.doi.org/10.1016/j.cub.2008.02.057

[56] Iacoboni, M., Molnar-Szakacs, I., Gallese, V., Buccino, G., Mazziotta, J.C. and Rizzolatti, G. (2005) Grasping the Intentions of Others with One’s Own Mirror Neuron System. PLoS Biology, 3, 529-535. http://dx.doi.org/10.1371/journal.pbio.0030079

[57] Press, C., Weiskopf, N. and Kilner, J.M. (2012) Dissociable Roles of Human Inferior Frontal Gyrus during Action Execution and Observation. NeuroImage, 60, 1671-1677. http://dx.doi.org/10.1016/j.neuroimage.2012.01.118

[58] Schubotz, R.I. and von Cramon, D.Y. (2001) Functional Organization of the Lateral Premotor Cortex: fMRI Reveals Different Regions Activated by Anticipation of Object Properties, Location and Speed. Cognitive Brain Research, 11, 97-112. http://dx.doi.org/10.1016/S0926-6410(00)00069-0

[59] Schubotz, R.I. and von Cramon, D.Y. (2002) Predicting Perceptual Events Activates Corresponding Motor Schemes in Lateral Premotor Cortex: An fMRI Study. Neuroimage, 15, 787-796. http://dx.doi.org/10.1006/nimg.2001.1043

[60] Schubotz, R.I. and von Cramon, D.Y. (2002) A Blueprint for Target Motion: fMRI Reveals Perceived Sequential Complexity to Modulate Premotor Cortex. Neuroimage, 16, 920-935. http://dx.doi.org/10.1006/nimg.2002.1183

[61] Hanakawa, T., Honda, M., Sawamoto, N., Okada, T., Yonekura, Y., Fukuyama, H., et al. (2002) The Role of Rostral Brodmann Area 6 in Mental-Operation Tasks: An Integrative Neuroimaging Approach. Cerebral Cortex, 12, 1157- 
1170. http://dx.doi.org/10.1093/cercor/12.11.1157

[62] Tanaka, S., Honda, M. and Sadato, N. (2005) Modality-Specific Cognitive Function of Medial and Lateral Human Brodmann Area 6. The Journal of Neuroscience, 25, 496-501. http://dx.doi.org/10.1523/JNEUROSCI.4324-04.2005

\section{Appendix A}

Table 1. Average oxy-Hb concentration changes under the novel contour shape condition during the correct responses.

\begin{tabular}{|c|c|c|c|c|c|c|c|c|c|c|c|c|c|}
\hline \multirow{2}{*}{$\begin{array}{l}\text { Novel } \\
\text { contour } \\
\text { shape }\end{array}$} & \multirow{2}{*}{ Channel } & \multicolumn{12}{|c|}{ Period } \\
\hline & & Pre & Fix 1 & $\begin{array}{c}\text { Targ } \\
1\end{array}$ & $\begin{array}{c}\text { Targ } \\
2\end{array}$ & $\begin{array}{c}\text { Targ } \\
3\end{array}$ & $\begin{array}{c}\text { Targ } \\
4\end{array}$ & $\begin{array}{c}\text { Targ } \\
5\end{array}$ & Fix 2 & Test & Conf & Post 1 & Post 2 \\
\hline \multirow[t]{10}{*}{ Easy } & 5 & 0 & 0.56 & 2.31 & 4.07 & 4.13 & 2.95 & 1.79 & 1.19 & 0.48 & 0.07 & -0.30 & 1.36 \\
\hline & 9 & 0 & 0.18 & 1.18 & 1.85 & 1.71 & 0.39 & -0.74 & -0.80 & -0.63 & -1.04 & -3.38 & -2.24 \\
\hline & 10 & 0 & 0.98 & 4.54 & 8.08 & 8.36 & 6.45 & 4.29 & 3.41 & 3.40 & 1.42 & -2.20 & 0.11 \\
\hline & 15 & 0 & -0.05 & 1.97 & 4.53 & 6.09 & 5.87 & 5.89 & 6.71 & 6.77 & 4.77 & 2.49 & 1.67 \\
\hline & 16 & 0 & 0.35 & 2.12 & 4.18 & 5.60 & 6.01 & 5.00 & 4.90 & 4.92 & 4.10 & 2.33 & 1.60 \\
\hline & 20 & 0 & 0.19 & 1.16 & 2.60 & 3.33 & 3.40 & 3.33 & 3.96 & 4.01 & 1.50 & -1.04 & -1.45 \\
\hline & 21 & 0 & 0.32 & 1.42 & 3.28 & 4.16 & 3.72 & 2.93 & 3.17 & 3.50 & 1.76 & -0.87 & -0.14 \\
\hline & 22 & 0 & 0.38 & 0.99 & 2.82 & 4.65 & 5.63 & 5.93 & 6.90 & 7.56 & 4.66 & 0.78 & 0.68 \\
\hline & 23 & 0 & 0.41 & 2.09 & 4.24 & 4.99 & 4.55 & 3.79 & 3.62 & 3.49 & 1.50 & -0.89 & -1.10 \\
\hline & 24 & 0 & 0.25 & 1.76 & 3.72 & 4.01 & 3.42 & 3.18 & 3.82 & 3.69 & 1.20 & -3.83 & -3.30 \\
\hline \multirow[t]{10}{*}{ Difficult } & 5 & 0 & 0.65 & 1.72 & 3.55 & 2.83 & -0.23 & -3.93 & -4.94 & -4.11 & -3.00 & -4.11 & -4.26 \\
\hline & 9 & 0 & -0.08 & 1.04 & 1.46 & 0.65 & -1.04 & -2.40 & -2.77 & -0.89 & 1.72 & -0.09 & 0.01 \\
\hline & 10 & 0 & 0.17 & -0.26 & -1.61 & -3.18 & -4.94 & -6.04 & -6.37 & -5.56 & -7.31 & -8.55 & -5.61 \\
\hline & 15 & 0 & 0.79 & 1.17 & 1.29 & 0.93 & -0.23 & -1.84 & -2.62 & -2.04 & -2.59 & -4.17 & -4.84 \\
\hline & 16 & 0 & 1.08 & 2.18 & 2.48 & 1.32 & -0.11 & -1.99 & -2.28 & -1.74 & -1.41 & -3.40 & -3.51 \\
\hline & 20 & 0 & -0.01 & 0.59 & 0.23 & -0.71 & -1.72 & -2.03 & -1.80 & -1.17 & -1.63 & -2.72 & -2.18 \\
\hline & 21 & 0 & 0.70 & 0.60 & -0.30 & -0.85 & -1.10 & -1.18 & -0.76 & -0.39 & -3.19 & -5.21 & -4.85 \\
\hline & 22 & 0 & 0.78 & 1.34 & 0.67 & -1.49 & -3.99 & -4.59 & -3.53 & -6.31 & -13.4 & -9.51 & -5.69 \\
\hline & 23 & 0 & 0.19 & 0.55 & 0.59 & -0.55 & -3.45 & -6.26 & -6.44 & -5.57 & -6.94 & -7.04 & -7.59 \\
\hline & 24 & 0 & 0.55 & 0.78 & -0.32 & -2.55 & -4.36 & -5.75 & -5.09 & -4.72 & -9.36 & -11.4 & -10.1 \\
\hline
\end{tabular}


Scientific Research Publishing (SCIRP) is one of the largest Open Access journal publishers. It is currently publishing more than 200 open access, online, peer-reviewed journals covering a wide range of academic disciplines. SCIRP serves the worldwide academic communities and contributes to the progress and application of science with its publication.

Other selected journals from SCIRP are listed as below. Submit your manuscript to us via either submit@scirp.org or Online Submission Portal.
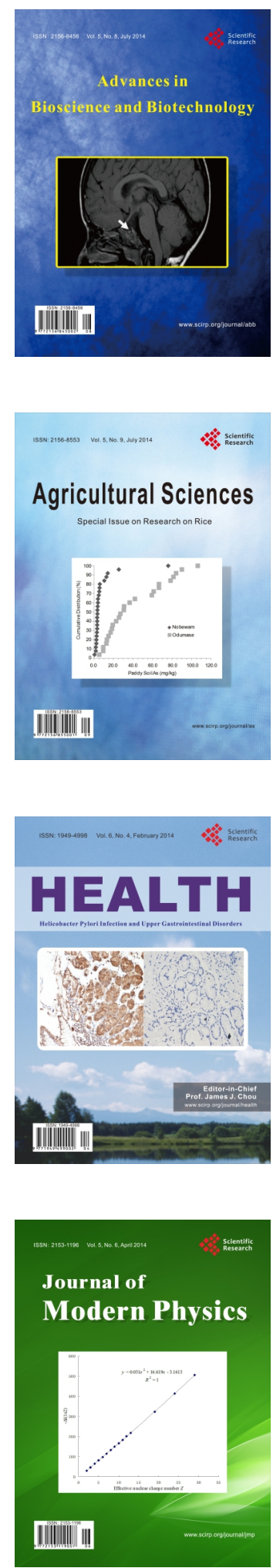
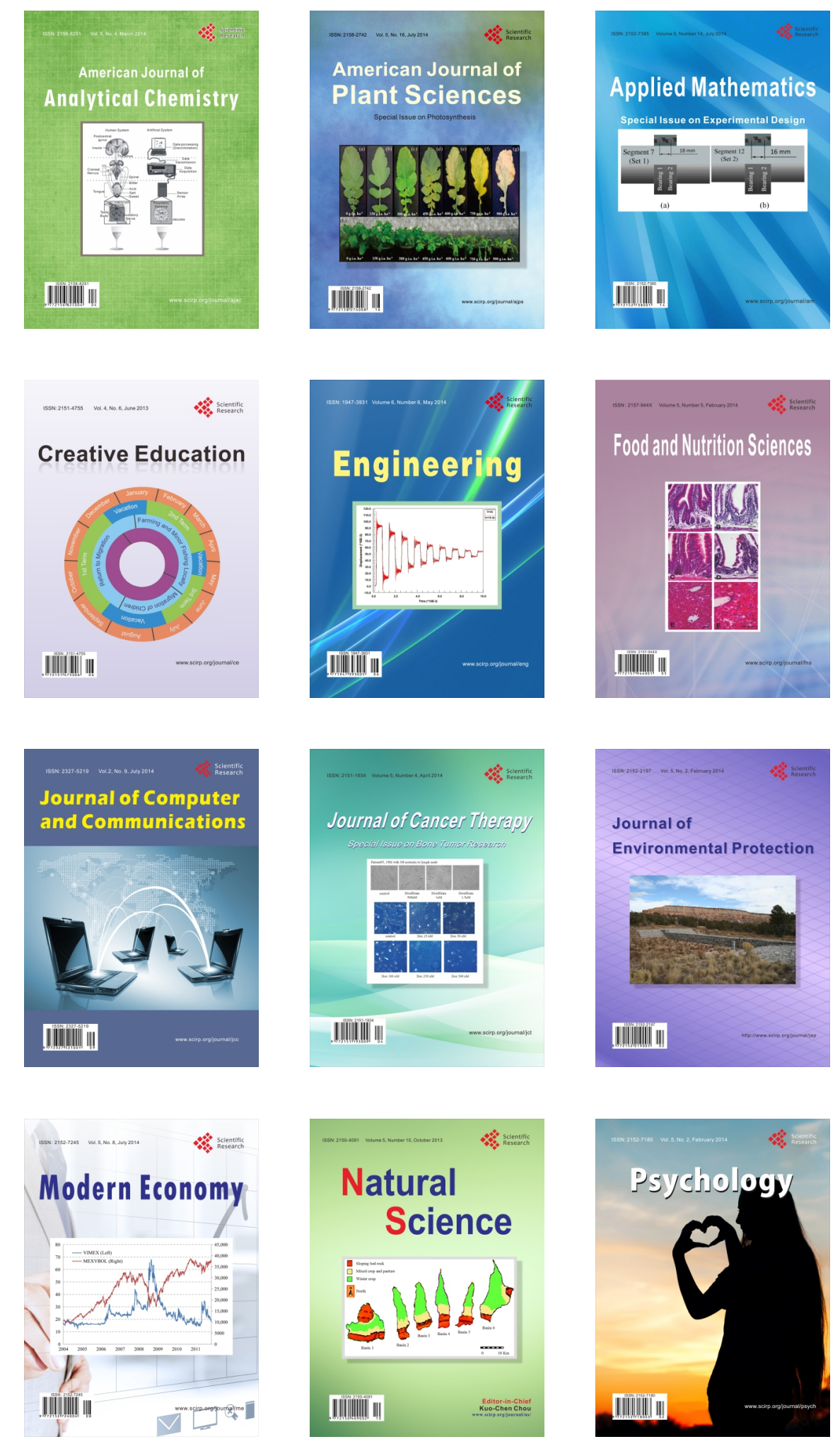